\title{
One kind of character sum modulo a prime $p$ and its recurrence formula
}

Wenpeng Zhang ${ }^{1,2}$ and Zhuoyu Chen ${ }^{2 *}$

${ }^{\text {"Correspondence: }}$

chenzymath@163.com

${ }^{2}$ School of Mathematics, Northwest University, Xi'an, P.R. China

Full list of author information is available at the end of the article

\begin{abstract}
The aim of this paper is to use an analytic method and the properties of the classical Gauss sums to research the computational problem of one kind of character sum of polynomials modulo an odd prime $p$ and obtain several meaningful third- and fourth-order linear recurrence formulae for them.

MSC: $11 \mathrm{~L} 10 ; 11 \mathrm{~L} 40$.

Keywords: The classical Gauss sums; Character sums of polynomials; Linear recurrence formula; Analytic method
\end{abstract}

\section{Introduction}

Let $q \geq 3$ be an integer, and let $\chi$ be a nonprincipal character mod $q$. Then for any integral coefficient polynomial $f(x)$, we define the character sum of the polynomial as

$$
N(\chi, f ; q)=\sum_{a=1}^{q} \chi(f(a)) .
$$

This sum plays an extremely significant role in analytic number theory, so they have aroused the interest and favor of a great deal of number theorists. A lot of works connected with $N(\chi, f ; q)$ can be found in [1-9] and [10-12]. In fact, the sums $N(\chi, f ; q)$ are a particular case of the general character sums of the polynomials

$$
\sum_{a=N+1}^{N+M} \chi(f(a))
$$

where $M$ and $N$ are any positive integers. If $q=p$ is an odd prime, then Weil (see [2] and [6]) obtained following significant conclusion:

Suppose that $\chi$ is a $q$ th-order character $\bmod p$ and $f(x)$ is not a perfect $q$ th power mod $p$. Then we have the estimate

$$
\sum_{x=N+1}^{N+M} \chi(f(x)) \ll p^{\frac{1}{2}} \ln p,
$$

where the constant in "«" depends only on the degree of $f(x)$. The estimate in (1) is the best possible.

(c) The Author(s) 2019. This article is distributed under the terms of the Creative Commons Attribution 4.0 International License (http://creativecommons.org/licenses/by/4.0/), which permits unrestricted use, distribution, and reproduction in any medium, provided you give appropriate credit to the original author(s) and the source, provide a link to the Creative Commons license, and indicate if changes were made. 
Now for any odd prime $p$ and any nonprincipal character $\chi$ mod $p$, we consider the following problem: for any positive integers $k$ and $h$, let

$$
M_{k}(h, \chi ; p)=\sum_{a_{1}=0}^{p-1} \sum_{a_{2}=0}^{p-1} \cdots \sum_{a_{k}=0}^{p-1} \chi\left(a_{1}^{h}+a_{2}^{h}+\cdots+a_{k}^{h}\right)
$$

and

$$
N_{k}(h, \chi ; p)=M_{k}(h, \chi ; p)+M_{k}(h, \bar{\chi} ; p) .
$$

We inquire if there exists an accurate computational formula for $N_{k}(h, \chi ; p)$ ?

About this contents, from our personal perspective, it appears that none had researched it yet; at least so far, we have not seen any related results before. The problem is meaningful, since it can help scholars to find out more exact information of the character sums.

In our paper, applying analytic methods and the properties of the classical Gauss sums, we researched the problem of calculating $N_{k}(h, \chi ; p)$ and obtained a significant linear recurrence formula for it. We will prove the following:

Theorem 1 Let $p$ be an odd prime, let $h$ be a positive integer, and let $\chi$ be any Dirichlet character $\bmod p$ such that $\chi^{h} \neq \chi_{0}$, the principal character $\bmod p$. Then for any positive integer $k$, we obtain the identity

$$
N_{k}(h, \chi ; p)=M_{k}(h, \chi ; p)=\sum_{a_{1}=0}^{p-1} \sum_{a_{2}=0}^{p-1} \cdots \sum_{a_{k}=0}^{p-1} \chi\left(a_{1}^{h}+a_{2}^{h}+\cdots+a_{k}^{h}\right)=0 .
$$

Theorem 2 Let $p$ be an odd prime with $p \equiv 1 \bmod 3$, and let $\chi$ be any third-order character $\bmod p$. Then we have the identities

$$
N_{1}(3, \chi ; p)=2(p-1) ; \quad N_{2}(3, \chi ; p)=(p-1) d ; \quad N_{3}(3, \chi ; p)=6 p(p-1)
$$

and

$$
N_{k}(3, \chi ; p)=3 p \cdot N_{k-2}(3, \chi ; p)+d p \cdot N_{k-3}(3, \chi ; p)
$$

for all integers $k \geq 4$, where $d$ is uniquely determined by $4 p=d^{2}+27 b^{2}$ and $d \equiv 1 \bmod 3$.

Theorem 3 Let $p=8 h+5$ be a prime, and let $\chi$ be any fourth-order character mod $p$. Then we have the identities

$$
\begin{aligned}
& N_{1}(4, \chi ; p)=2(p-1), \quad N_{2}(4, \chi ; p)=-4(p-1) \alpha, \\
& N_{3}(4, \chi ; p)=2(p-1)\left(p-2 \alpha^{2}\right)
\end{aligned}
$$

and

$$
N_{k+4}(4, \chi ; p)=-2 p N_{k+2}(4, \chi ; p)+8 p \alpha N_{k+1}(4, \chi ; p)-p\left(9 p-4 \alpha^{2}\right) N_{k}(4, \chi ; p)
$$


for all $k \geq 0$, where $N_{0}(4, \chi ; p)=0, \alpha=\sum_{a=1}^{\frac{p-1}{2}}\left(\frac{a+\bar{a}}{p}\right),\left(\frac{*}{p}\right)$ denotes the Legendre symbol mod $p$, and $a \bar{a} \equiv 1 \bmod p$.

Theorem 4 Let $p=8 h+1$ be a prime, and let $\chi$ be a fourth-order character mod $p$. Then we have the identities

$$
N_{1}(4, \chi ; p)=2(p-1), \quad N_{2}(4, \chi ; p)=4(p-1) \alpha, \quad N_{3}(4, \chi ; p)=2(p-1)\left(2 \alpha^{2}-p\right)
$$

and

$$
N_{k+4}(4, \chi ; p)=6 p N_{k+2}(4, \chi ; p)+8 p \alpha N_{k+1}(4, \chi ; p)+p\left(4 \alpha^{2}-p\right) N_{k}(4, \chi ; p)
$$

for all integers $k \geq 0$, where $N_{0}(4, \chi ; p)=0$.

From the methods of proving the theorems, we can also infer the following:

Corollary 1 Let $p$ be a prime with $p \equiv 1 \bmod 3$, and let $\chi$ be a third-order character $\bmod p$. Then for any integer $k \geq 0$, we have the identity

$$
\left|\sum_{a=0}^{p-1} \sum_{b=0}^{p-1} \sum_{c=0}^{p-1} \sum_{d=0}^{p-1} \chi\left(a^{3}+b^{3}+c^{3}+d^{3}\right)\right|=(p-1) p \cdot \sqrt{9 p+4 d^{2}}
$$

Corollary 2 Let $p=4 k+1$ be an odd prime, and let $\chi$ be a fourth-order character mod $p$. Then we have the identity

$$
\left|\sum_{a=0}^{p-1} \sum_{b=0}^{p-1} \chi\left(a^{4}+b^{4}\right)\right|=2(p-1) \sqrt{p} .
$$

Corollary 3 Let $p=4 k+1$ be an odd prime, and let $\chi$ be a fourth-order character mod $p$. Then we have the identity

$$
\left|\sum_{a=0}^{p-1} \sum_{b=0}^{p-1} \sum_{c=0}^{p-1} \chi\left(a^{4}+b^{4}+c^{4}\right)\right|=(p-1) p .
$$

Corollary 4 Let $p=8 k+5$ be an odd prime, and let $\chi$ be a fourth-order character mod $p$. Then we have the identity

$$
\left|\sum_{a=0}^{p-1} \sum_{b=0}^{p-1} \sum_{c=0}^{p-1} \sum_{d=0}^{p-1} \chi\left(a^{4}+b^{4}+c^{4}+d^{4}\right)\right|=4(p-1) p \cdot \sqrt{p+8 \alpha^{2}} .
$$

Corollary 5 Let $p=8 k+1$ be an odd prime, and let $\chi$ be a fourth-order character mod $p$. Then we have the identity

$$
\left|\sum_{a=0}^{p-1} \sum_{b=0}^{p-1} \sum_{c=0}^{p-1} \sum_{d=0}^{p-1} \chi\left(a^{4}+b^{4}+c^{4}+d^{4}\right)\right|=4(p-1) p \cdot \sqrt{9 p+16 \alpha^{2}} .
$$




\section{Several lemmas}

In this section, we give several lemmas, which are essential in the proofs of our theorems. Hereinafter, we are going to use some properties of the classical Gauss sums, which can be found in some analytic number theory books, such as [13]; so we will not repeat them here. For convenience, first, we give the definition of the classical Gauss sums $\tau(\chi)$ as follows: for any integer $q>1$, let $\chi$ be any Dirichlet character $\bmod q$. Then the famous Gauss sum $\tau(\chi)$ is defined as

$$
\tau(\chi)=\sum_{a=1}^{q} \chi(a) e\left(\frac{a}{q}\right)
$$

where $e(y)=e^{2 \pi i y}$. With this mark, we have the following:

Lemma 1 Given $p$ be an odd prime with $p \equiv 1 \bmod 3$, and let $\psi$ be any third-order character $\bmod p$. Then we have the identity

$$
\tau^{3}(\psi)+\tau^{3}(\bar{\psi})=d p
$$

where $d$ is uniquely determined by $4 p=d^{2}+27 b^{2}$ and $d \equiv 1 \bmod 3$.

Proof See Lemma 3 of [9] or references [14] and [10].

Lemma 2 Given $p$ be an odd prime with $p \equiv 1 \bmod 3$, and for any integer $b$ with $(b, p)=1$, let $U(b, p)=\sum_{a=0}^{p-1} e\left(\frac{b a^{3}}{p}\right)$. Then we have the identity

$$
U^{3}(b, p)=d p+3 p \cdot U(b, p)
$$

where $d$ is the same as in Lemma 1.

Proof Let $\chi$ be any third-order character $\bmod p$. Then $\chi^{2}=\bar{\chi}$, and from Lemma 1 and the properties of Gauss sums we have

$$
\begin{aligned}
U(b, p) & =\sum_{a=0}^{p-1} e\left(\frac{b a^{3}}{p}\right)=1+\sum_{a=1}^{p-1} e\left(\frac{b a^{3}}{p}\right) \\
& =1+\sum_{a=1}^{p-1}(1+\chi(a)+\bar{\chi}(a)) e\left(\frac{b a}{p}\right) \\
& =\sum_{a=0}^{p-1} e\left(\frac{b a}{p}\right)+\sum_{a=1}^{p-1} \chi(a) e\left(\frac{b a}{p}\right)+\sum_{a=1}^{p-1} \bar{\chi}(a) e\left(\frac{b a}{p}\right) \\
& =\bar{\chi}(b) \tau(\chi)+\chi(b) \tau(\bar{\chi}) .
\end{aligned}
$$

Note that $\chi^{3}=\chi_{0}$ and $\tau(\chi) \tau(\bar{\chi})=p$, so from (2) we immediately infer that

$$
\begin{aligned}
U^{3}(b, p) & =(\bar{\chi}(b) \tau(\chi)+\chi(b) \tau(\bar{\chi}))^{3} \\
& =\tau^{3}(\chi)+3 \tau(\chi) \tau(\bar{\chi})(\bar{\chi}(b) \tau(\chi)+\chi(b) \tau(\bar{\chi}))+\tau^{3}(\bar{\chi})
\end{aligned}
$$




$$
=\tau^{3}(\chi)+\tau^{3}(\bar{\chi})+3 p \cdot U(b, p)=d p+3 p \cdot U(b, p) .
$$

This proves Lemma 2.

Lemma 3 Given $p$ be an odd prime with $p \equiv 1 \bmod 4$, and let $\psi$ be any fourth-order character $\bmod p$. Then we have the identity

$$
\tau^{2}(\psi)+\tau^{2}(\bar{\psi})=\sqrt{p} \cdot \sum_{a=1}^{p-1}\left(\frac{a+\bar{a}}{p}\right)=2 \sqrt{p} \cdot \alpha,
$$

where $\alpha=\sum_{a=1}^{\frac{p-1}{2}}\left(\frac{a+\bar{a}}{p}\right),\left(\frac{*}{p}\right)$ denotes the Legendre symbol mod $p$, and $\bar{a}$ denotes the multiplicative inverse of a $\bmod p$, that is, $a \bar{a} \equiv 1 \bmod p$.

Proof In fact, this is Lemma 2.2 in [15]. Therefore we omit its proof.

Lemma 4 Let $p$ be an odd prime with $p \equiv 1 \bmod 4$, and for any integer $b$ with $(b, p)=1$, let $A(b, p)=\sum_{a=0}^{p-1} e\left(\frac{b a^{4}}{p}\right)$. Then we have the identities

$$
A^{4}(b, p)=2 p(C(p)+2) A^{2}(b, p)+8 p \alpha A(b, p)+4 p \alpha^{2}-C^{2}(p) p^{2}
$$

where $C(p)=-3$ if $p=8 k+5$ and $C(p)=1$ if $p=8 k+1$.

Proof Let $\psi$ be a fourth-order character $\bmod p$. Then $\psi^{2}=\chi_{2}$ (the Legendre symbol mod $p)$, and by the properties of Gauss sums we get

$$
\begin{aligned}
A(b, p) & =\sum_{a=0}^{p-1} e\left(\frac{b a^{4}}{p}\right)=1+\sum_{a=1}^{p-1} e\left(\frac{b a^{4}}{p}\right) \\
& =1+\sum_{a=1}^{p-1}\left(1+\psi(a)+\chi_{2}(a)+\bar{\psi}(a)\right) e\left(\frac{b a}{p}\right) \\
& =\sum_{a=0}^{p-1} e\left(\frac{b a}{p}\right)+\sum_{a=1}^{p-1} \psi(a) e\left(\frac{b a}{p}\right)+\sum_{a=1}^{p-1} \chi_{2}(a) e\left(\frac{b a}{p}\right)+\sum_{a=1}^{p-1} \bar{\psi}(a) e\left(\frac{b a}{p}\right) \\
& =\chi_{2}(b) \tau\left(\chi_{2}\right)+\bar{\psi}(b) \tau(\psi)+\psi(b) \tau(\bar{\psi}) .
\end{aligned}
$$

Note that $\psi^{2}=\bar{\psi}^{2}=\chi_{2}, \tau(\psi) \tau(\bar{\psi})=\psi(-1) \tau(\psi) \overline{\tau(\psi)}=\psi(-1) p$, and $\tau\left(\chi_{2}\right)=\sqrt{p}$. Combining (3) and Lemma 2, we have

$$
\begin{aligned}
A^{2}(b, p)= & \left(\chi_{2}(b) \tau\left(\chi_{2}\right)+\bar{\psi}(b) \tau(\psi)+\psi(b) \tau(\bar{\psi})\right)^{2} \\
= & p+2 \chi_{2}(b) \sqrt{p}(\bar{\psi}(b) \tau(\psi)+\psi(b) \tau(\bar{\psi}))+\chi_{2}(b)\left(\tau^{2}(\psi)+\tau^{2}(\bar{\psi})\right) \\
& +2 \tau(\psi) \tau(\bar{\psi}) \\
= & p+2 \chi_{2}(b) \sqrt{p} A(b, p)-2 p+\chi_{2}(b) 2 \sqrt{p} \cdot \alpha+\psi(-1) 2 p \\
= & C(p) p+2 \chi_{2}(b) \sqrt{p} A(b, p)+\chi_{2}(b) 2 \sqrt{p} \cdot \alpha,
\end{aligned}
$$

where $C(p)=-3$ if $p=8 k+5$ and $C(p)=1$ if $p=8 k+1$. 
Now, according to (4), we have

$$
\begin{aligned}
\left(A^{2}(b, p)-C(p) p\right)^{2} & =A^{4}(b, p)-2 C(p) p A^{2}(b, p)+C^{2}(p) p^{2} \\
& =(2 \sqrt{p} A(b, p)+2 \sqrt{p} \cdot \alpha)^{2}=4 p\left(A^{2}(b, p)+2 \alpha A(b, p)+\alpha^{2}\right) .
\end{aligned}
$$

Applying (5), we immediately deduce that

$$
A^{4}(b, p)=2 p(C(p)+2) A^{2}(b, p)+8 p \alpha A(b, p)+4 p \alpha^{2}-C^{2}(p) p^{2}
$$

This proves Lemma 4.

Lemma 5 Let $p$ be an odd prime with $p \equiv 1 \bmod 3$, and let $\chi$ be any third-order character $\bmod p$. Then we have the identities

$$
M_{1}(3, \chi ; p)=p-1 ; \quad M_{2}(3, \chi ; p)=\frac{p-1}{p} \cdot \tau^{3}(\chi) ; \quad M_{3}(3, \chi ; p)=3 p(p-1)
$$

and

$$
M_{k}(3, \chi ; p)=3 p \cdot M_{k-2}(3, \chi ; p)+d p \cdot M_{k-3}(3, \chi ; p)
$$

for all integers $k \geq 4$, where $d$ is the same as in Lemma 1 .

Proof It is obvious that $M_{1}(3, \chi ; p)=p-1$. According to (2) and the properties of Gauss sums, we get

$$
\begin{aligned}
M_{2}(3, \chi ; p) & =\frac{1}{\tau(\bar{\chi})} \sum_{b=1}^{p-1} \bar{\chi}(b) \sum_{c=0}^{p-1} \sum_{d=0}^{p-1} e\left(\frac{b\left(c^{3}+d^{3}\right)}{p}\right) \\
& =\frac{1}{\tau(\bar{\chi})} \sum_{b=1}^{p-1} \bar{\chi}(b)(\bar{\chi}(b) \tau(\chi)+\chi(b) \tau(\bar{\chi}))^{2} \\
& =\frac{1}{\tau(\bar{\chi})} \sum_{b=1}^{p-1} \bar{\chi}(b)\left(\bar{\chi}^{2}(b) \tau^{2}(\chi)+2 p+\chi^{2}(b) \tau^{2}(\bar{\chi})\right) \\
& =\frac{\tau^{2}(\chi)}{\tau(\bar{\chi})} \cdot(p-1)=\frac{p-1}{p} \cdot \tau^{3}(\chi), \\
M_{3}(3, \chi ; p) & =\frac{1}{\tau(\bar{\chi})} \sum_{b=1}^{p-1} \bar{\chi}(b) \sum_{c=0}^{p-1} \sum_{d=0}^{p-1} \sum_{e=0}^{p-1} e\left(\frac{b\left(c^{3}+d^{3}+e^{3}\right)}{p}\right) \\
& =\frac{1}{\tau(\bar{\chi})} \sum_{b=1}^{p-1} \bar{\chi}(b)(\bar{\chi}(b) \tau(\chi)+\chi(b) \tau(\bar{\chi}))^{3} \\
& =\frac{1}{\tau(\bar{\chi})} \sum_{b=1}^{p-1} \bar{\chi}(b)\left(\tau^{3}(\chi)+3 p U(b, p)+\tau^{3}(\bar{\chi})\right)=3 p(p-1) .
\end{aligned}
$$


For any integer $k \geq 4$, according to Lemma 1 , we have

$$
\begin{aligned}
M_{k}(3, \chi ; p) & =\frac{1}{\tau(\bar{\chi})} \sum_{b=1}^{p-1} \bar{\chi}(b)\left(\sum_{a=0}^{p-1} e\left(\frac{b a^{3}}{p}\right)\right)^{k} \\
& =\frac{1}{\tau(\bar{\chi})} \sum_{b=1}^{p-1} \bar{\chi}(b) U^{k-3}(b, p)\left(\tau^{3}(\chi)+\tau^{3}(\bar{\chi})+3 p U(b, p)\right) \\
& =\frac{3 p}{\tau(\bar{\chi})} \sum_{b=1}^{p-1} \bar{\chi}(b) U^{k-2}(b, p)+\frac{d p}{\tau(\bar{\chi})} \sum_{b=1}^{p-1} \bar{\chi}(b) U^{k-3}(b, p) \\
& =3 p M_{k-2}(3, \chi ; p)+d p \cdot M_{k-3}(3, \chi ; p) .
\end{aligned}
$$

Now Lemma 5 follows from (6), (7), and (8).

Lemma 6 Let $p=8 h+5$ be a prime, and let $\chi$ be any fourth-order character mod $p$. Then we have the identities

$$
\begin{aligned}
& M_{1}(4, \chi ; p)=p-1 ; \\
& M_{2}(4, \chi ; p)=2(p-1) \sqrt{p} \cdot \frac{\tau(\chi)}{\tau(\bar{\chi})}=-\frac{2(p-1)}{\sqrt{p}} \cdot \tau^{2}(\chi) \\
& M_{3}(4, \chi ; p)=(p-1) \frac{\tau^{3}(\chi)}{\tau(\bar{\chi})}=-\frac{p-1}{p} \cdot \tau^{4}(\chi) .
\end{aligned}
$$

For every integer $k \geq 0$, we obtain the fourth-order linear recurrence formula

$$
M_{k+4}(4, \chi ; p)=-2 p M_{k+2}(4, \chi ; p)+8 p \alpha M_{k+1}(4, \chi ; p)-p\left(9 p-4 \alpha^{2}\right) M_{k}(4, \chi ; p)
$$

where $M_{0}(4, \chi ; p)=0$, and $\alpha$ is the same as in Lemma 3.

Proof Let $p$ be an odd prime with $p=8 h+5$, and let $\chi$ be any fourth-order character mod $p$. Then this time we obtain $C(p)=-3$. For any integer $k \geq 0$, according to the properties of Gauss sums and Lemma 4, we get

$$
\begin{aligned}
M_{k+4}(4, \chi ; p) & =\frac{1}{\tau(\bar{\chi})} \sum_{x_{1}=0}^{p-1} \sum_{x_{2}=0}^{p-1} \cdots \sum_{x_{k+4}=0}^{p-1} \sum_{b=1}^{p-1} \bar{\chi}(b) e\left(\frac{b\left(x_{1}^{4}+x_{2}^{2}+\cdots+x_{k+4}^{4}\right)}{p}\right) \\
& =\frac{1}{\tau(\bar{\chi})} \sum_{b=1}^{p-1} \bar{\chi}(b)\left(\sum_{x=0}^{p-1} e\left(\frac{b x^{4}}{p}\right)\right)^{k+4}=\frac{1}{\tau(\bar{\chi})} \sum_{b=1}^{p-1} \bar{\chi}(b) A^{k+4}(b, p) \\
& =\frac{1}{\tau(\bar{\chi})} \sum_{b=1}^{p-1} \bar{\chi}(b) A^{k}(b, p)\left(-2 p A^{2}(b, p)+8 p \alpha A(b, p)-p\left(9 p-4 \alpha^{2}\right)\right) \\
& =-2 p M_{k+2}(4, \chi ; p)+8 p \alpha M_{k+1}(4, \chi ; p)-p\left(9 p-4 \alpha^{2}\right) M_{k}(4, \chi ; p),
\end{aligned}
$$

where $M_{0}(4, \chi ; p)=0$. 
Suppose that $\chi$ is a fourth-order character $\bmod p$. Then we get

$$
M_{1}(4, \chi, p)=\sum_{a=0}^{p-1} \chi\left(a^{4}\right)=\sum_{a=0}^{p-1} \chi^{4}(a)=p-1 .
$$

Note that $\bar{\chi}(b)\left(\frac{b}{p}\right)=\chi(b)$, so from (4) and (3) we get

$$
\begin{aligned}
M_{2}(4, \chi ; p) & =\frac{1}{\tau(\bar{\chi})} \sum_{b=1}^{p-1} \bar{\chi}(b)\left(\sum_{x=0}^{p-1} e\left(\frac{b x^{4}}{p}\right)\right)^{2} \\
& =\frac{1}{\tau(\bar{\chi})} \sum_{b=1}^{p-1} \bar{\chi}(b)\left(-3 p+\chi_{2}(b) 2 \sqrt{p} A(b, p)+\chi_{2}(b) 2 \sqrt{p} \alpha\right) \\
& =\frac{2 \sqrt{p}}{\tau(\bar{\chi})} \sum_{b=1}^{p-1} \chi(b) A(b, p) \\
& =\frac{2 \sqrt{p}}{\tau(\bar{\chi})} \sum_{b=1}^{p-1} \chi(b)\left(\chi_{2}(b) \sqrt{p}+\bar{\chi}(b) \tau(\chi)+\chi(b) \tau(\bar{\chi})\right) \\
& =-\frac{2(p-1)}{\sqrt{p}} \cdot \tau^{2}(\chi) .
\end{aligned}
$$

In the same way, applying (3) and the orthogonality of the characters mod $p$, we also have

$$
\begin{aligned}
M_{3}(4, \chi ; p) & =\frac{1}{\tau(\bar{\chi})} \sum_{b=1}^{p-1} \bar{\chi}(b)\left(\sum_{x=0}^{p-1} e\left(\frac{b x^{4}}{p}\right)\right)^{3} \\
& =\frac{1}{\tau(\bar{\chi})} \sum_{b=1}^{p-1} \bar{\chi}(b)\left(\chi_{2}(b) \sqrt{p}+\bar{\chi}(b) \tau(\chi)+\chi(b) \tau(\bar{\chi})\right)^{3} \\
& =\frac{3 p}{\tau(\bar{\chi})} \sum_{b=1}^{p-1} \bar{\chi}(b) \chi(b) \tau(\bar{\chi})+\frac{1}{\tau(\bar{\chi})} \sum_{b=1}^{p-1} \tau^{3}(\chi)+\frac{3}{\tau(\bar{\chi})} \sum_{b=1}^{p-1} \tau(\chi) \tau^{2}(\bar{\chi}) \\
& =-\frac{p-1}{p} \cdot \tau^{4}(\chi) .
\end{aligned}
$$

Combining (9)-(12), we immediately obtain Lemma 6.

Lemma 7 Let $p=8 h+1$ be a prime, and let $\chi$ be any fourth-order character $\bmod p$. Then we have the identities

$$
\begin{aligned}
& M_{1}(4, \chi ; p)=p-1 \\
& M_{2}(4, \chi ; p)=2(p-1) \sqrt{p} \cdot \frac{\tau(\chi)}{\tau(\bar{\chi})}=\frac{2(p-1)}{\sqrt{p}} \cdot \tau^{2}(\chi) \\
& M_{3}(4, \chi ; p)=\frac{p-1}{p} \cdot \tau^{4}(\chi) .
\end{aligned}
$$

For every integer $k \geq 0$, we have the fourth-order linear recurrence formula

$$
M_{k+4}(4, \chi ; p)=6 p M_{k+2}(4, \chi ; p)+8 p \alpha M_{k+1}(4, \chi ; p)+p\left(4 \alpha^{2}-p\right) M_{k}(4, \chi ; p) .
$$


Proof If $p=8 h+1$, then from Lemma 4 we have

$$
\begin{aligned}
M_{k+4}(4, \chi ; p) & =\frac{1}{\tau(\bar{\chi})} \sum_{x_{1}=0}^{p-1} \sum_{x_{2}=0}^{p-1} \ldots \sum_{x_{k+4}=0}^{p-1} \sum_{b=1}^{p-1} \bar{\chi}(b) e\left(\frac{b\left(x_{1}^{4}+x_{2}^{2}+\cdots+x_{k+4}^{4}\right)}{p}\right) \\
& =\frac{1}{\tau(\bar{\chi})} \sum_{b=1}^{p-1} \bar{\chi}(b) A^{k}(b, p)\left(6 p A^{2}(b, p)+8 p \alpha A(b, p)+p\left(4 \alpha^{2}-p\right)\right) \\
& =6 p M_{k+2}(4, \chi ; p)+8 p \alpha M_{k+1}(4, \chi ; p)+p\left(4 \alpha^{2}-p\right) M_{k}(4, \chi ; p) .
\end{aligned}
$$

It is not complicated to prove that

$$
M_{1}(4, \chi ; p)=\sum_{x=0}^{p-1} \chi\left(x^{4}\right)=\sum_{x=0}^{p-1} \chi^{4}(x)=p-1
$$

Note that $\bar{\chi}(b)\left(\frac{b}{p}\right)=\chi(b), \tau(\chi) \tau(\bar{\chi})=p$. By the method of proving (11) we have

$$
\begin{aligned}
M_{2}(4, \chi ; p) & =\frac{1}{\tau(\bar{\chi})} \sum_{b=1}^{p-1} \bar{\chi}(b)\left(\sum_{x=0}^{p-1} e\left(\frac{b x^{4}}{p}\right)\right)^{2} \\
& =\frac{2 \sqrt{p} \cdot \tau(\chi)}{\tau(\bar{\chi})} \sum_{a=1}^{p-1} 1=\frac{2(p-1)}{\sqrt{p}} \cdot \tau^{2}(\chi) .
\end{aligned}
$$

Similarly, combined with the method of proving (12), we also get

$$
\begin{aligned}
M_{3}(4, \chi ; p) & =\frac{1}{\tau(\bar{\chi})} \sum_{b=1}^{p-1} \bar{\chi}(b)\left(\sum_{x=0}^{p-1} e\left(\frac{b x^{4}}{p}\right)\right)^{3} \\
& =(p-1) \cdot \frac{\tau^{3}(\chi)}{\tau(\bar{\chi})}=\frac{p-1}{p} \cdot \tau^{4}(\chi) .
\end{aligned}
$$

Now Lemma 7 follows from (13)-(16).

\section{Proofs of the theorems}

In this section, we complete the proofs of our theorems. First of all, we prove Theorem 1 by mathematical induction. If $k=1$, then note that $\chi^{h} \neq \chi_{0}$. According to the properties of character sums mod $p$, we obtain the identity

$$
\sum_{a=0}^{p-1} \chi\left(a^{h}\right)=\sum_{a=1}^{p-1} \chi^{h}(a)=0
$$

Suppose that the conclusion holds for an integer $k=m \geq 1$, that is,

$$
\sum_{a_{1}=0}^{p-1} \sum_{a_{2}=0}^{p-1} \cdots \sum_{a_{m}=0}^{p-1} \chi\left(a_{1}^{h}+a_{2}^{h}+\cdots+a_{m}^{h}\right)=0
$$


Then for $k=m+1$, combining (17) and (18) with the properties of the complete residue system $\bmod p$, we have

$$
\begin{aligned}
& \sum_{a_{1}=0}^{p-1} \sum_{a_{2}=0}^{p-1} \cdots \sum_{a_{m}=0}^{p-1} \sum_{a_{m+1}=0}^{p-1} \chi\left(a_{1}^{h}+a_{2}^{h}+\cdots+a_{m}^{h}+a_{m+1}^{h}\right) \\
& =\sum_{a_{1}=0}^{p-1} \sum_{a_{2}=0}^{p-1} \cdots \sum_{a_{m}=0}^{p-1} \chi\left(a_{1}^{h}+a_{2}^{h}+\cdots+a_{m}^{h}\right) \\
& \quad+\sum_{a_{1}=0}^{p-1} \sum_{a_{2}=0}^{p-1} \cdots \sum_{a_{m}=0}^{p-1} \sum_{a_{m+1}=1}^{p-1} \chi\left(a_{1}^{h}+a_{2}^{h}+\cdots+a_{m}^{h}+a_{m+1}^{h}\right) \\
& =\sum_{a_{1}=0}^{p-1} \sum_{a_{2}=0}^{p-1} \cdots \sum_{a_{m}=0}^{p-1} \chi\left(a_{1}^{h}+a_{2}^{h}+\cdots+a_{m}^{h}+1\right) \sum_{a_{m+1}=1}^{p-1} \chi^{h}\left(a_{m+1}\right) \\
& =0 .
\end{aligned}
$$

Thus the conclusion is also correct for $k=m+1$. This proves Theorem 1 .

Now, we are going to prove Theorem 2 . From Lemmas 1 and 5 , noting that by definition $N_{k}(h, \chi ; p)=M_{k}(h, \chi ; p)+M_{k}(h, \bar{\chi} ; p)$, we get

$$
\begin{aligned}
& N_{1}(3, \chi ; p)=2(p-1), \quad N_{3}(3, \chi ; p)=6 p(p-1), \\
& N_{2}(3, \chi ; p)=\frac{p-1}{p} \cdot \tau^{3}(\chi)+\frac{p-1}{p} \cdot \tau^{3}(\bar{\chi})=d(p-1)
\end{aligned}
$$

and

$$
N_{k}(3, \chi ; p)=3 p \cdot N_{k-2}(3, \chi ; p)+d p \cdot N_{k-3}(3, \chi ; p)
$$

for all integers $k \geq 4$. This proves Theorem 2 .

Suppose that $p$ is an odd prime with $p=8 h+5$ and that $\chi$ is a fourth-order character $\bmod p$. Then applying Lemmas 3 and 6 , we have

$$
\begin{aligned}
& N_{1}(4, \chi ; p)=2(p-1) ; \\
& N_{2}(4, \chi ; p)=-\frac{2(p-1)}{\sqrt{p}} \cdot \tau^{2}(\chi)-\frac{2(p-1)}{\sqrt{p}} \cdot \tau^{2}(\bar{\chi})=-4(p-1) \cdot \alpha .
\end{aligned}
$$

By the identities $\overline{\tau(\chi)}^{2}=\tau^{2}(\bar{\chi}), \tau^{2}(\chi) \tau^{2}(\bar{\chi})=\tau^{2}(\chi) \overline{\tau(\chi)}^{2}=p^{2}$, and

$$
\tau^{4}(\chi)+\tau^{4}(\bar{\chi})=\left(\tau^{2}(\chi)+\tau^{2}(\bar{\chi})\right)^{2}-2 \tau^{2}(\chi) \tau^{2}(\bar{\chi})=4 p \alpha^{2}-2 p^{2},
$$

we have

$$
\begin{aligned}
N_{3}(4, \chi ; p) & =-\frac{p-1}{p} \cdot \tau^{4}(\chi)-\frac{p-1}{p} \cdot \tau^{4}(\bar{\chi}) \\
& =-\frac{p-1}{p}\left(4 p \alpha^{2}-2 p^{2}\right)=2\left(p-2 \alpha^{2}\right)(p-1) .
\end{aligned}
$$


For every integer $k \geq 0$, we get the fourth-order linear recurrence formula

$$
\begin{aligned}
& N_{k+4}(4, \chi ; p) \\
& \quad=-2 p N_{k+2}(4, \chi ; p)+8 p \alpha N_{k+1}(4, \chi ; p)-p\left(9 p-4 \alpha^{2}\right) N_{k}(4, \chi ; p),
\end{aligned}
$$

where $N_{0}(4, \chi ; p)=0$, and $\alpha$ is the same as in Lemma 3 .

Now Theorem 3 follows from (19)-(22).

In the same way, using Lemmas 3 and 7, we can also deduce Theorem 4.

To prove Corollary 1 , applying Lemma 5 , we get

$$
M_{4}(3, \chi ; p)=3(p-1) \tau^{3}(\chi)+d p(p-1) .
$$

Then from this formula and Lemma 1 , by the identities $|\tau(\chi)|=\sqrt{p}$ and $\overline{\tau^{3}(\chi)}=\tau^{3}(\bar{\chi})$ we have

$$
\begin{aligned}
& \left|\sum_{a=0}^{p-1} \sum_{b=0}^{p-1} \sum_{c=0}^{p-1} \sum_{c=0}^{p-1} \chi\left(a^{3}+b^{3}+c^{3}+d^{3}\right)\right| \\
& \quad=\left|3(p-1) \tau^{3}(\chi)+d p(p-1)\right| \\
& \quad=(p-1)\left|3 \tau^{3}(\chi)+d p\right|=(p-1)\left[9 p^{3}+3 d p\left(\tau^{3}(\chi)+\tau^{3}(\bar{\chi})\right)+d^{2} p^{2}\right]^{\frac{1}{2}} \\
& \quad=(p-1)\left[9 p^{3}+3 d^{2} p^{2}+d^{2} p^{2}\right]^{\frac{1}{2}}=(p-1) p \cdot \sqrt{9 p+4 d^{2}} .
\end{aligned}
$$

This proves Corollary 1.

Corollaries 2 and 3 follow from Lemma 6 and the identity $|\tau(\chi)|=\sqrt{p}$.

Next, we are going to prove Corollary 4. If $p=8 k+5$, then noting that $\overline{\tau(\chi)}^{2}=\tau^{2}(\bar{\chi})$ and $|\alpha+\beta|^{2}=|\alpha|^{2}+\alpha \bar{\beta}+\bar{\alpha} \beta+|\beta|^{2}$, from Lemmas 6 and 3 we have

$$
\begin{aligned}
\left|M_{4}(4, \chi ; p)\right| & =\left|4 \sqrt{p}(p-1) \tau^{2}(\chi)+8 p \alpha(p-1)\right| \\
& =4 \sqrt{p}(p-1)\left|\tau^{2}(\chi)+2 \sqrt{p} \alpha\right| \\
& =4 \sqrt{p}(p-1)\left|p^{2}+4 p \alpha^{2}+2 \sqrt{p} \alpha\left(\tau^{2}(\chi)+\tau^{2}(\bar{\chi})\right)\right|^{\frac{1}{2}} \\
& =4 \sqrt{p}(p-1)\left|p^{2}+8 p \alpha^{2}\right|^{\frac{1}{2}}=4 p(p-1) \cdot \sqrt{p+8 \alpha^{2}} .
\end{aligned}
$$

In the same way, if $p=8 k+1$, then from Lemmas 7 and 3 , by the method of proving (23) we have

$$
\begin{aligned}
\left|M_{4}(4, \chi ; p)\right| & =\left|12 \sqrt{p}(p-1) \tau^{2}(\chi)+8 p \alpha(p-1)\right| \\
& =4 \sqrt{p}(p-1)\left|3 \tau^{2}(\chi)+2 \sqrt{p} \alpha\right| \\
& =4 \sqrt{p}(p-1)\left|9 p^{2}+4 p \alpha^{2}+6 \sqrt{p} \alpha\left(\tau^{2}(\chi)+\tau^{2}(\bar{\chi})\right)\right|^{\frac{1}{2}} \\
& =4 \sqrt{p}(p-1)\left|9 p^{2}+16 p \alpha^{2}\right|^{\frac{1}{2}}=4 p(p-1) \cdot \sqrt{9 p+16 \alpha^{2}} .
\end{aligned}
$$

Combining (23) and (24), we complete the proofs of Corollaries 4 and 5. 


\section{Acknowledgements}

The authors would like to thank the editors and referees for their very helpful and detailed comments, which have significantly improved the presentation of this paper.

Funding

This work is supported by the N. S. F. (11771351) and (11826205) of P. R. China.

\section{Competing interests}

The authors declare that there are no conflicts of interest regarding the publication of this paper.

\section{Authors' contributions}

Both authors have equally contributed to this work. Both authors read and approved the final manuscript.

\section{Author details}

'School of Science, Xi'an Technological University, Xi'an, P.R. China. ${ }^{2}$ School of Mathematics, Northwest University, Xi'an, P.R. China.

\section{Publisher's Note}

Springer Nature remains neutral with regard to jurisdictional claims in published maps and institutional affiliations.

Received: 18 February 2019 Accepted: 29 March 2019 Published online: 05 April 2019

\section{References}

1. Burgess, D.A.: On character sums and primitive roots. Proc. Lond. Math. Soc. 12, 179-192 (1962)

2. Burgess, D.A.: On Dirichlet characters of polynomials. Proc. Lond. Math. Soc. 13, 537-548 (1963)

3. Granville, A., Soundararajan, K.: Large character sums: pretentious characters and the Pólya-Vinogradov theorem. J. Am. Math. Soc. 20, 357-384 (2007)

4. Zhang, W.P., Yi, Y.: On Dirichlet characters of polynomials. Bull. Lond. Math. Soc. 34, 469-473 (2002)

5. Zhang, W.P., Yao, W.L.: A note on the Dirichlet characters of polynomials. Acta Arith. 115, 225-229 (2004)

6. Weil, A.: On some exponential sums. Proc. Natl. Acad. Sci. USA 34, 204-207 (1948)

7. Bourgain, J., Garaev, M.Z., Konyagin, S.V., Shparlinski, I.E.: On the hidden shifted power problem. SIAM J. Comput. 41 1524-1557 (2012)

8. Zhang, W.P., Liu, H.N.: On the general Gauss sums and their fourth power mean. Osaka J. Math. 42, 189-199 (2005)

9. Zhang, W.P.: On the number of the solutions of one kind congruence equation mod p. J. Northwest Univ. Nat. Sci. 46, 313-316 (2016)

10. Zhang, W.P., Hu, J.Y.: The number of solutions of the diagonal cubic congruence equation mod p. Math. Rep. 20 , 73-80 (2018)

11. Zhang, H., Zhang, W.P.: The fourth power mean of two-term exponential sums and its application. Math. Rep. 19, 75-83 (2017)

12. Chen, L., Hu, J.Y.: A linear recurrence formula involving cubic Gauss sums and Kloosterman sums. Acta Math. Sinica (Chin. Ser.) 61, 67-72 (2018)

13. Apostol, T.M.: Introduction to Analytic Number Theory. Springer, New York (1976)

14. Berndt, B.C., Evans, R.J.: The determination of Gauss sums. Bull. Am. Math. Soc. 5, 107-128 (1981)

15. Chen, Z.Y., Zhang, W.P.: On the fourth-order linear recurrence formula related to classical Gauss sums. Open Math. 15, $1251-1255(2017)$

\section{Submit your manuscript to a SpringerOpen ${ }^{\odot}$ journal and benefit from:}

- Convenient online submission

Rigorous peer review

- Open access: articles freely available online

- High visibility within the field

- Retaining the copyright to your article

Submit your next manuscript at $\boldsymbol{~ s p r i n g e r o p e n . c o m ~}$ 